\title{
Uso y abuso de los catéteres intravenosos en las plantas de hospitalización convencional
}

\author{
S. HERRERO GÁMIZ, P. MARTÍNEZ DE ALBORNOZ TORRENTE, B. NAVARRO \\ VIDAL, E. SALVADOR ÁLVAREZ, J. VILLACORTA PÉREZ, R. SAN JUAN \\ GARRIDO, J. M. AGUADO GARCÍA \\ Unidad de Enfermedades Infecciosas. Hospital Universitario 12 de Octubre. Madrid
}

\author{
USE AND ABUSE OF INTRAVENOUS CATHETERS IN CONVENTIO- \\ NAL HOSPITAL WARDS
}

\section{RESUMEN}

Antecedentes: Existe escasa información acerca del uso de catéteres venosos (CVs) en unidades de hospitalización convencionales y la incidencia bacteriemia relacionada con catéteres venosos (BRCV) en dichas unidades.

Objetivos: Evaluar el uso de catéteres venosos (CVs) en pacientes ingresados en plantas convencionales de un hospital general y las consecuencias en términos de BRCV.

Métodos: Se evaluó durante una semana el uso de catéteres venosos y la densidad de incidencia de BRCV en pacientes adultos ingresados en los servicios de hospitalización del hospital 12 de Octubre de Madrid.

Resultados: Se analizaron las historias clínicas de 731 pacientes (284 en servicios médicos y 447 en servicios quirúrgicos), de los cuales 338 $(46,2 \%)$ eran portadores en ese momento de un $\mathrm{CV}$ periférico y 63 $(8,6 \%)$ de un CV central. La media de días de colocación de un CV central fue globalmente de 11,5 (IC 95\% 5,57-17,42), 28,3 en los servicios médicos días y 8,32 en los servicios quirúrgicos $(\mathrm{p}=0,2)$. En el $27,7 \%$ de los pacientes con CVs la única indicación para el uso de un CV fue la administración intravenosa de antibióticos a pesar de que el paciente toleraba por vía oral y en un $30 \%$ de las ocasiones no había ninguna especificación en la historia que permitiera aclarar cuál era la indicación para la colocación del CV. Se detectó BRCV en 12/401 pacientes (3\%). La densidad de incidencia de BRC en CV centrales fue de 8,28 por cada 1.000 días de catéter.

Conclusiones: Existen claros aspectos que deben mejorarse en cuanto a la prevención de la BRC, fundamentalmente respecto al ajuste de las indicaciones, el tiempo que permanecen los catéteres centrales implantados $y$, sobre todo, en cuanto a las oportunidades perdidas de retirar el catéter secuenciando la medicación a vía oral.

PALABRAS CLAVE: Catéter intravenoso. Infección. Bacteriemia. Uso. Prevención

\section{ABSTRACT}

Background: Information regarding the use intravenous catheters (IVC) in conventional hospital units and its consequence in terms of intravenous catéter-related bacteremia (ICRB) is scarce.

Objectives: To evaluate the use of IVC in patients admitted in conventional wards of a general hospital and to measure IVCRB incidence in such patients.

Methods: We evaluated during one week IVC use in adult patients admitted in 12 de Octubre Hospital and we calculated la incidence densitity of ICRB.

Results: We evaluated the clinical charts of 731 patients (284 from medical wards and 447 from surgical wards), of which 338 (46.2\%) had a peripheral VC inserted and $63(8.6 \%)$ a central IVC. Central IVC had been inserted for a mean time 11.5 days globally (CI 95\% 5.57-17.42), being 28.3 in medical wards and 8.32 days in surgical wards $(p=0.2)$. In $27.7 \%$ of the patients with IVC intravenous antimicrobials was the only reason for the use of such catheters in spite of adequate oral tolerance in $30 \%$ of the patients with central IVC an specific note explaining the reason for implanting such catheter was lacking in the clinical chart. IVCRB was detected in 12/401 patients (3\%). The incidence density of IVCRB in central IVC was 8.28 per 1000 catheter-days.

Conclusions: There are some aspects that could be clearly improved regarding the prevention of IVCRB, mostly in the indications, the excess of time those catheters are kept implanted and in the lost chances for catheter withdrawal when switch-therapy could be performed.

KEY WORDS: Intravenous catheter. Infection. Bacteremia. Use. Prevention.

Herrero Gámiz S, Martínez de Albornoz Torrente P, Navarro Vidal B, Salvador Álvarez, E, Villacorta Pérez, J, San Juan Garrido R, Aguado García JM. Uso y abuso de los catéteres intravenosos en las plantas de hospitalización convencional. An Med Interna (Madrid) 2006; 23: 475-477.

\section{INTRODUCCIÓN}

Una de las causas más importantes de infección nosocomial es la derivada del uso de un catéter venoso (CV), que constituye la principal causa de bacteriemia nosocomial y está relacionada con una alta morbilidad y mortalidad (1). Según datos del proyecto EPINE, el 50\% de los pacientes hospitalizados en nuestros hospitales son portadores de un CV (2). Muchos de estos CVs podrían no ser imprescindibles o se podría acortar el tiempo durante el cual permanecen coloca-

Trabajo aceptado: 18 de mayo de 2006

Correspondencia: Rafael San Juan. Unidad de Enfermedades Infecciosas. Hospital Universitario 12 de Octubre. Edificio Materno-Infantil. Planta 6a A. Avda. de Córdoba km 5,400. 28041 Madrid. e-mail: rafasjg@yahoo.es 
dos, lo que permitiría reducir la incidencia de bacteriemia secundaria que se estima es de 2,5 a 3,4 episodios/1.000 enfermos (3) y las complicaciones secundarias de estas bacteriemias (endocarditis, espondilitis, etc.) pueden ser devastadoras para el paciente.

La mayoría de estudios relacionados con el uso de CVs en los hospitales incluyen pacientes ingresados en unidades de cuidados intensivos o pacientes oncohematológicos que son portadores de CVs de larga duración con una elevada incidencia de complicaciones infecciosas, pero hay menos estudios referidos exclusivamente a pacientes ingresados en plantas de hospitalización convencional. En este estudio quisimos evaluar cuál era la prevalencia del uso de CVs en pacientes ingresados en plantas convencionales de un hospital general, cuál era el riesgo de sufrir bacteriemia secundaria en estos pacientes y cuál era el porcentaje de pacientes en los que se hubiera podido acortar el tiempo de utilización del CV.

\section{MATERIAL Y MÉTODOS}

Para ello de se realizó un estudio de prevalencia del empleo de CVs en plantas de hospitalización convencional del Hospital Universitario 12 de Octubre, un centro de nivel terciario con 1.429 camas. Se incluyeron en el estudio pacientes adultos (mayores de 18 años) ingresados en los servicios médicos y quirúrgicos del hospital. Se excluyeron del estudio los pacientes ingresados en los servicios de Oncología, Hematología y UCI.

La recogida de información se realizó durante una semana (del 28 de noviembre al 3 de diciembre de 2004). Se recogieron datos de las historias clínicas y de los informes microbiológicos (cultivo de la punta del CV y hemocultivos). Las variables analizadas fueron: edad, sexo, enfermedades de base, días de ingreso, sala de ingreso (médica o quirúrgica), indicación de uso del CV, tipo de CV (central o periférico), lugar de inserción, duración de la colocación del CV, tolerancia o no por vía oral, administración o no de antibióticos por el CV y presencia o no de otros dispositivos cardiovasculares (marcapasos, prótesis valvulares o vasculares).

Se consideró que la bacteriemia estaba relacionada con el catéter (BRC) si se cumplía alguna de las siguientes condiciones: a) en caso de retirada del CV, cuando el microorganismo aislado en los hemocultivos era el mismo que se aislaba de la punta del CV (> 15 ufc en cultivo semicuantitativo, según técnica de Maki); y b) en caso de no retirada del CV: cuando existían hemocultivos positivos para un microorganismo de frecuente presencia en bacteriemias asociadas a catéter (Staphylococcus coagulasa negativos, S. aureus, Corynebacterium spp., Propionibacterium spp. etc.) y no existía ningún otro foco probable de bacteriemia.

Las variables continuas se expresaron con la media y la desviación estándar (DS) para aquellas variables con distribución normal. Las variables discretas se expresaron en porcentajes.

El análisis estadístico se llevó a cabo utilizando el paquete estadístico SPSS 12.0. Para la comparación de variables continuas se utilizó el test de Student y el test de Chi-cuadrado o el test exacto de Fischer para la comparación de proporciones. El límite para la asignación de significación bilateral de los tests fue una $\mathrm{p}<0,05$.

\section{RESULTADOS}

Se evaluaron 731 pacientes ingresados en plantas de hospitalización convencional: 284 pacientes estaban ingresados en servicios médicos $(38,9 \%)$ y 447 en servicios quirúrgicos $(61,1 \%)$. La media de edad fue de 59 años (IC95\% 56,8-62,6), con un intervalo entre 18 y 93 años.

De los 731 pacientes evaluados 401 (55\%) eran portadores en ese momento de un CV. El porcentaje de pacientes con CV en los servicios médicos fue del $34,2 \%$ y en los servicios quirúrgicos del 65,8\% ( $\mathrm{p}=0,005$ ) (Fig. 1). El 15,7\% de los pacientes (63/401) eran portadores de CV centrales (incluyendo CV centrales transitorios y de acceso periférico) y el 84,3\% (338/401) tenían CV periféricos. Globalmente, la prevalencia del uso de CVs periféricos fue de un $46,2 \%$ y de CVs centrales del 8.6\%. Los CVs centrales supusieron el 7,3\% del total de accesos vasculares en los servicios médicos comparado con el 20,1\% en los servicios quirúrgicos ( $\mathrm{p}<0,0018)$.

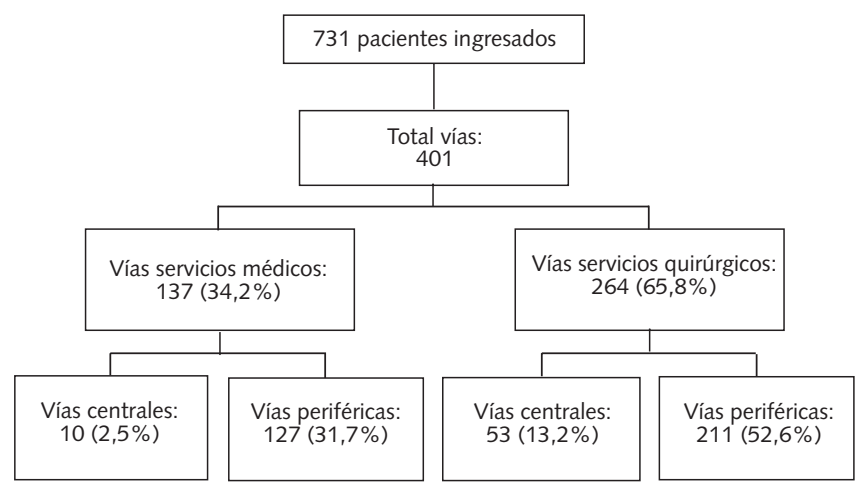

Fig. 1. Distribución de pacientes según el tipo de servicio (médico/quirúrgico) y de catéter venoso (central/periférico).

La media de días de colocación de un CV fue globalmente de 11,5 (IC 95\% 5,57-17,42). En los servicios médicos fue de 28,30 días (mediana 9 días, intervalo 0-180 días) y en los servicios quirúrgicos de 8,32 días (mediana 6 días, intervalo 0-54 días), aunque las diferencias no fieron estadísticamente significativas $(\mathrm{p}=0,2)$.

La indicación más frecuente para el uso de un CV fue la administración de fármacos (en el $87 \%$ de los CVs centrales y en el $69,5 \%$ de los CVs periféricos) o de sueroterapia (en el $54 \%$ de los CVs centrales y en el $31,1 \%$ de los CVs periféricos). Es de destacar que en el $27,7 \%$ de los pacientes (111/401) la única indicación para el uso de un CV fue la administración intravenosa de antibióticos a pesar de que el paciente toleraba por vía oral y podría haber recibido otros antibióticos de administración oral que tuvieran una eficacia similar. Por último, en un 30\% de las ocasiones no había ninguna especificación en la historia que permitiera aclarar cuál era la indicación para la colocación del CV.

Durante el período de estudio se detectó BRC probable en 12 de los 401 pacientes portadores de CV, lo que supone una prevalencia del 3\%. Los microorganismos aislados fueron: $\mathrm{S}$. coagulasa negativo (57\%), S. aureus $(28,6 \%)$, Candida albicans $(7,2 \%)$ y Klebsiella $(7,2 \%)$. La prevalencia de BRC fue 
similar en los servicios médicos y quirúrgicos $(3,6$ vs. 2,6\% (p $>0,05$ ), pero significativamente mayor en los pacientes con $\mathrm{CV}$ central que en los pacientes con $\mathrm{CV}$ periférico $(9,5$ vs. $1,8 \% ; \mathrm{p}=0,005)$. La densidad de incidencia de BRC en CV centrales fue de 8,28 por cada 1.000 días de catéter: 10,6 episodios por 1.000 días de catéter en los servicios médicos y 6,8 episodios por 1.000 días de catéter en los servicios quirúrgi$\cos (\mathrm{p}>0,05)$.

\section{DISCUSIÓN}

Este estudio de prevalencia muestra que más de la mitad de los pacientes ingresados en plantas de hospitalización convencional de un hospital de tercer nivel en España tienen colocado un CV. Es de destacar que el 8,6\% de los pacientes ingresados en estas plantas son portadores de CVs centrales. Los CVs están colocados durante periodos prolongados de tiempo (media 11,5 días, IC95\% 5,57-17,42), un tiempo mayor que la media encontrada en el estudio del National Nosocomial Infections Surbeillance System (NNISS) que fue de 8 días (3), lo que supone un riesgo elevado de que el paciente sufra complicaciones infecciosas locales o sistémicas, fundamentalmente bacteriemia.

La utilización de CVs, especialmente de CVs centrales, fue más frecuente en servicios quirúrgicos que en servicios médicos, pero la media de días de colocación del catéter fue superior en los servicios médicos que en los quirúrgicos (28,30 vs. 8,32 días respectivamente) lo que pudo contribuir a que la incidencia acumulada de BRC fuera mayor en los servicios médicos (10,6 vs. 6,8 episodios por 1.000 días de catéter), aunque la diferencia no resultó ser estadísticamente significativa.

La prevalencia de BRC fue del 3\% y llegó a ser del 9,5\% en pacientes portadores de un $\mathrm{CV}$ central, una prevalencia similar a la descrita en otros estudios españoles (4). La inci- dencia acumulada de BRC fue 8,28 episodios por 1.000 días de catéter, una incidencia elevada si comparamos con otras series $(5,6)$ especialmente teniendo en cuenta que una de las limitaciones de este estudio es que no se realizó un seguimiento prospectivo de los pacientes lo que pudo hacer que el riesgo de BRC fuera infraestimado.

A pesar de que este trabajo no está diseñado para evaluar la adecuación del uso de los CVs, algunos datos del estudio señalan que probablemente se podría prescindir del empleo de CVs en un buen número pacientes. Es de destacar que en el 30\% de los pacientes con CV no existía ninguna referencia en la historia clínica del paciente que explicara el motivo de la colocación del mismo, ni existían datos clínicos aparentes que aconsejaran su uso. Además el 27,7\% de los pacientes tenían colocado un $\mathrm{CV}$ exclusivamente para recibir antibióticos intravenosos, a pesar de que toleraban por vía oral. La posibilidad de terapia secuencial antibiótica por vía oral podría haber obviado el uso de $\mathrm{CV}$ en más de la cuarta parte de nuestros pacientes, de hecho y esta política de control de antibióticos ha demostrado su utilidad en nuestro propio hospital (7).

En resumen, este trabajo intenta alertar, especialmente a aquellos médicos en formación, sobre la gran relevancia que plantea el uso abusivo y generalizado de CVs en las plantas de hospitalización convencional de nuestros hospitales. Existen claros aspectos que deben mejorarse en cuanto a la prevención de la BRC, fundamentalmente respecto al ajuste de las indicaciones de implantar una vía central, al tiempo que permanecen los catéteres centrales implantados (especialmente en servicios médicos) y, sobre todo, en cuanto a las oportunidades perdidas de retirar el catéter secuenciando la medicación a vía oral, hecho que, según este estudio, sería posible en más de una cuarta parte de los pacientes que reciben antibióticos parenterales. En este sentido la evaluación de la indicación de catéter debería ser revisada diariamente para evitar un uso excesivo e innecesario de los accesos vasculares.

\section{Bibliografía}

1. O'Grady NP, Alexander M, Dellinger EP, et al. Guidelines for the prevention of intravascular catheter-related infections. Centers for Disease Control and Prevention. MMWR Recomm Rep 2002; 51: 1-29. 2.

2. Asensio A, Cantón R, Vaque J, Roselló J, Arribas JL. Etiología de la infección nosocomial en hospitales españoles. (EPINE, 1990-1999). Med Clin (Barc) 2002; 118: 725-30

3. Center for Disease Control and Prevention NNIS System. National Nosocomial Infections Surveillance (NNIS) System report, data summary from January 1992-April 2000.Issued June 2000. Am J Infect Control 2000; 28: 429-48.

4. León C, Ariza J. Conferencia de consenso SEIMC-SEMICYUC. Enferm Infecc Microbiol Clin 2004; 22: 92-101
5. Richards MJ, Edwards JR, Culver DH, et al. Nosocomial infections in combined medical-surgical intensive care units in the United States. Infect Control Hosp Epidemiol 2000; 21: 510-5.

6. Bouza E, San Juan R, Muñoz P, et al. A European perspective on intravascular catheter-related infections: report on the microbiology workload, aetiology and antimicrobial susceptibility (ESGNI-005 Study) Clin Microbiol Infect 2004; 10: 838-842 .

7. López Medrano F, San Juan R, Serrano O, Chaves F, Lumbreras F, Lizasoain M, et al. PACTA: efecto de un programa no impositivo de control y asesoramiento del tratamiento antibiótico sobre la disminución de los costes y el descenso de ciertas infecciones nosocomiales. Enferm Infecc Microbiol Clin 2005; 23: 186-190. 